\title{
Case Report \\ Native Pulmonic Valve Endocarditis due to Mycobacterium fortuitum: A Case Report and Literature Review
}

\author{
Aaron M. Mulhall ${ }^{1}$ and Renee S. Hebbeler-Clark ${ }^{1,2}$ \\ ${ }^{1}$ Division of Pulmonary, Critical Care, and Sleep Medicine, University of Cincinnati Medical Center, 231 Albert Sabin Way, \\ Cincinnati, OH 45267, USA \\ ${ }^{2}$ Division of Infectious Diseases, University of Cincinnati Medical Center, 231 Albert Sabin Way, Cincinnati, OH 45267, USA \\ Correspondence should be addressed to Aaron M. Mulhall; ammulh01@gmail.com
}

Received 8 May 2015; Revised 1 June 2015; Accepted 2 June 2015

Academic Editor: Daniela M. Cirillo

Copyright (c) 2015 A. M. Mulhall and R. S. Hebbeler-Clark. This is an open access article distributed under the Creative Commons Attribution License, which permits unrestricted use, distribution, and reproduction in any medium, provided the original work is properly cited.

Endocarditis secondary to Mycobacterium fortuitum is a rare entity often involving prosthetic valves and rarely native valves. Pulmonic valve endocarditis secondary to any organism is rare. We report the first case of native pulmonic valve endocarditis secondary to $M$. fortuitum and a literature review of native valve $M$. fortuitum endocarditis.

\section{Introduction}

Mycobacterium fortuitum, a member of rapidly growing nontuberculous mycobacteria, is a well-known cause of skin and soft tissue infections and postsurgical wound infections [1]. Sporadic cases of endocarditis associated with this organism have been reported, mostly involving prosthetic valves. Isolated pulmonic valve endocarditis caused by any organism is rare, making up about $2 \%$ of all cases of infective endocarditis [2]. Nontuberculous mycobacteria pulmonic valve endocarditis has never been reported in the literature. Here, we describe a case of native pulmonic valve endocarditis secondary to $M$. fortuitum as well as a review of the literature.

\section{Case Presentation}

A 64-year-old female with a history of ventilator dependent respiratory failure secondary to end-stage chronic obstructive pulmonary disease (COPD) and bioprosthetic mitral valve replacement 8 years ago was admitted to the medical intensive care unit (MICU) for worsening respiratory failure from pulmonary edema and multifocal pneumonia secondary to Escherichia coli. She was diuresed and completed a course of antibiotics with ceftriaxone. A transesophageal echocardiogram (TEE) was performed during this admission revealing severe prosthetic mitral valve stenosis, mildly elevated pulmonary artery pressures, a normal tricuspid valve, and no vegetation on any valve. She was ultimately transferred to a long term postacute care hospital. Over the following month, the patient continued to decline in clinical status, and further investigation was undertaken. Rapid-growing mycobacterium was isolated from three cultures for mycobacteria from a tracheal aspirate. Interferon Gamma Release Assay (IGRA) and $M$. tuberculosis complex polymerase chain reaction (PCR) of a tracheal aspirate were negative. Rapidgrowing mycobacterium was isolated from multiple blood cultures from a peripherally inserted central catheter (PICC) and from peripheral venipuncture. $M$. fortuitum was the organism identified in all cultures. Empiric antibiotic therapy was initiated with amikacin, imipenem, and clarithromycin; and the PICC line was removed. The resistance-pattern confirmed that the isolate was sensitive to amikacin and imipenem, but resistant to clarithromycin. The patient was ultimately readmitted to the MICU for hypotension and concerns for overdiuresis and volume depletion. A transthoracic echocardiogram (TTE) was performed (40 days after the previous TTE and 12 days after initiation of antibiotics) revealing 


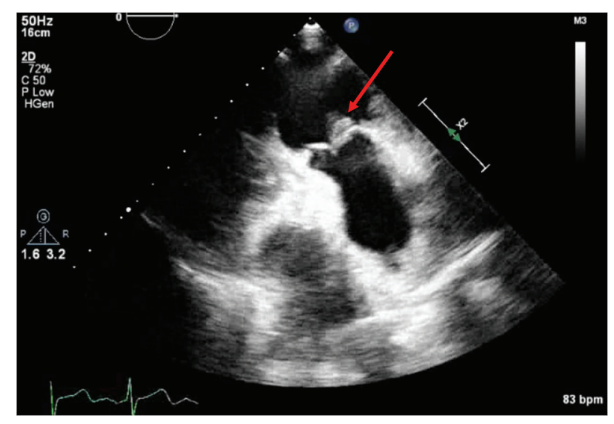

FIgURE 1: Transthoracic echocardiogram showing a $10 \mathrm{~mm} \times 11 \mathrm{~mm}$ vegetation (arrow) on the ventricular aspect of the pulmonic valve.

a new $10 \mathrm{~mm} \times 11 \mathrm{~mm}$ vegetation on the ventricular aspect of the pulmonic valve, severe tricuspid regurgitation, and severe prosthetic valve mitral stenosis (Figure 1). The patient was not a candidate for pulmonic valve replacement and medical management was continued. Given the grim prognosis, the patient ultimately decided to stop antibiotic therapy and entered hospice where she ultimately passed away. The patient completed 16 days of antibiotics at the time she was discharged to hospice.

\section{Discussion}

Mycobacterium fortuitum is a ubiquitous organism that can be found in soil, dust, and tap water and is well known for causing skin infections and postsurgical wound infections [1]. It belongs to the family of rapid-growing nontuberculous mycobacteria, meaning that it grows in culture within one week. Infections with this organism are often a result of direct inoculation, usually as a result of posttraumatic infections, postsurgical wound infections, and catheter-associated infections. Disseminated infections mostly occur in immunodeficient patients [3].

Endocarditis caused by this organism is uncommon, mostly affecting patients with prosthetic valves [4-6]. Endocarditis of native cardiac valves due to $M$. fortuitum is a rare occurrence, with only a small number of cases being reported [7-12] (Table 1). Upon review of the nine cases of native valve $M$. fortuitum endocarditis (including our own) the mean age was 24.4 years old (range $0.5-64$ years); the population was $66 \%$ female; valves infected were $44 \%$ aortic, $44 \%$ tricuspid (one patient with both aortic and tricuspid vegetation), 22\% mitral (one patient with both aortic and mitral vegetation), and $11 \%$ pulmonic; $56 \%$ had either an open or percutaneous cardiac procedure as the inciting event inoculating the patient and $22 \%$ were infected secondary to intravenous drug use; $22 \%$ received a valve replacement or removal of implanted cardiac hardware; and $44 \%$ of patients were alive at the time of their respective case report publication (in our case, the patient entered hospice).

There is only anecdotal evidence for the recommended treatment of severe disseminated $M$. fortuitum infections. All reviewed cases of native valve endocarditis secondary to this organism were treated with parenteral amikacin plus two of the following: fluoroquinolone, cefoxitin, TMP/SMX, or a macrolide. These agents should be initiated and continued for several weeks (most cases were treated for 6 weeks) until clinical improvement was identified and then transitioned to oral therapy with two effective agents for 6-12 months.

Our patient's isolate was sensitive to amikacin and imipenem, but resistant to clarithromycin. It is difficult to determine if the vegetation arose during antibiotic therapy, as she was only treated for 12 days prior to finding this vegetation. Antibiotic resistance and bacterial control were not major concerns based on the isolate resistance-pattern. We believe that she likely developed this vegetation in the previous month when she was clinically declining from an unclear etiology. Unfortunately, in this case, we could not confirm, by tissue diagnosis, that the vegetation was due to $M$. fortuitum. This is because the patient was not a surgical candidate for pulmonic valve replacement and the family declined a postmortem examination. While it is possible that the vegetation was not due to $M$. fortuitum, it is highly unlikely in the setting of new cardiac valve vegetation and persistently positive blood cultures for M. fortuitum.

There are a couple of potential mechanisms to explain how our patient became infected. One possibility is that our patient had airway colonization with $M$. fortuitum related to her end-stage COPD and the organism entered her bloodstream, subsequently seeding her pulmonic valve. $M$. fortuitum is commonly isolated from respiratory cultures in patients with underlying lung disease including COPD, pulmonary fibrosis, bronchiectasis, and prior pulmonary tuberculosis infection [13].

It is thought that the mycobacteria in the lungs gains access to the bloodstream via translocation. Previous studies of $M$. tuberculosis suggest that the organism is ingested by alveolar macrophages and then is transported by these macrophages and peripheral blood monocytes across the alveolar wall into the bloodstream. More recent evidence suggests that mycobacteria can also invade type II alveolar epithelial cells and translocate across the epithelial wall into the bloodstream [14]. Another potential mechanism is that our patient acquired $M$. fortuitum by a catheter-associated bloodstream infection that subsequently seeded her lungs and pulmonic valve. The propensity of $M$. fortuitum to cause catheter-related infections, its resistance to a number of antibiotics, and its association with tap water and water distribution centers have some relation with its ability to form biofilms [15].

Our case is the first reported case of $M$. fortuitum native valve endocarditis affecting the pulmonic valve. It is also the first reported case to have the organism isolated from a pulmonary source (tracheal aspirate) as well as the bloodstream. Even with antibiotics this disease is highly fatal and source control with valve replacement/removal of infectious hardware greatly improves survival [12]. The extremely poor prognosis associated with this disease reinforces the need to consider endocarditis in any patient with Mycobacterium bacteremia, regardless of species. 


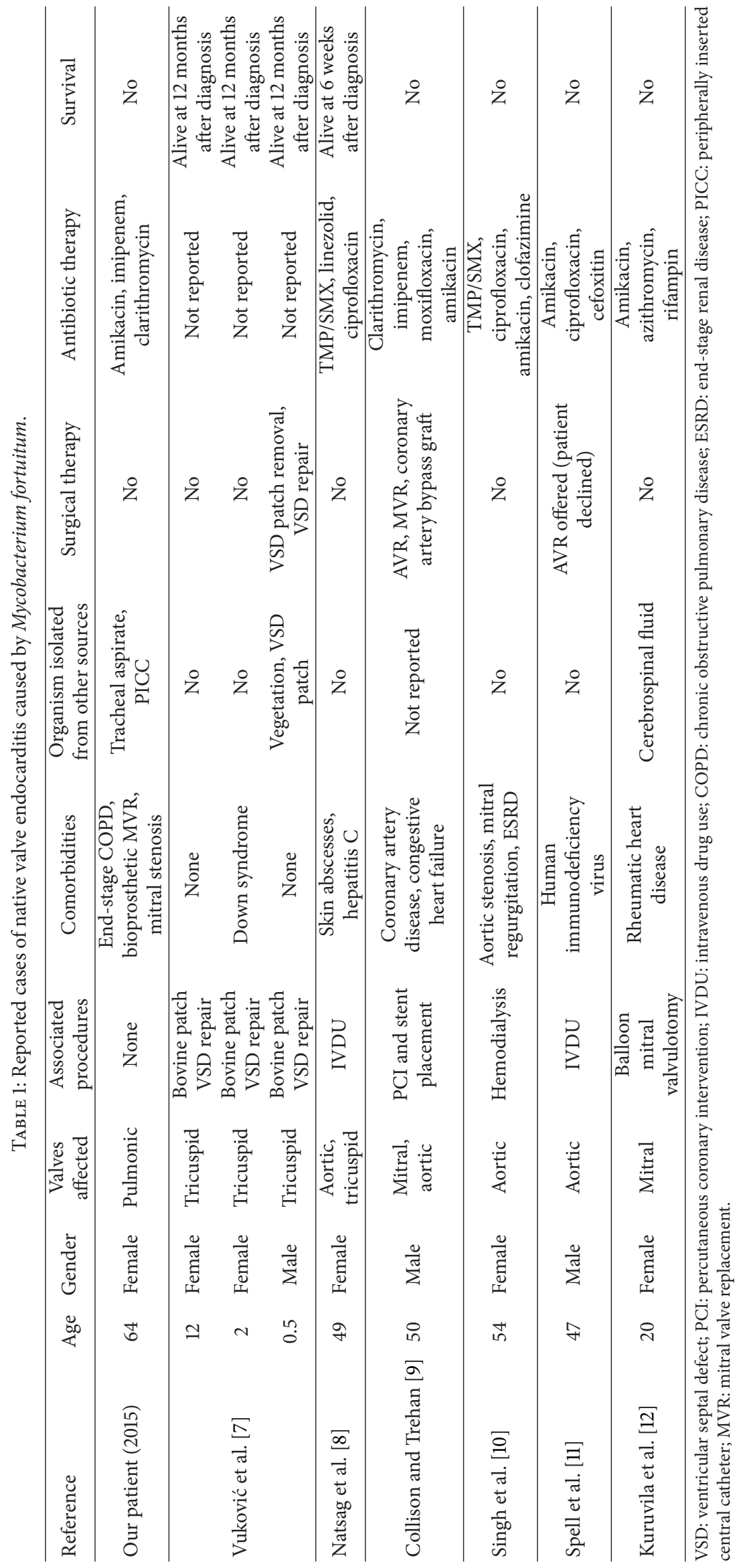




\section{Conflict of Interests}

The authors declare that there is no conflict of interests regarding the publication of this paper.

\section{References}

[1] D. E. Griffith, T. Aksamit, B. A. Brown-Elliott et al., "An official ATS/IDSA statement: diagnosis, treatment, and prevention of nontuberculous mycobacterial diseases," American Journal of Respiratory and Critical Care Medicine, vol. 175, no. 4, pp. 367416, 2007.

[2] K. Nishida, O. Fukuyama, and D. S. Nakamura, "Pulmonary valve endocarditis caused by right ventricular outflow obstruction in association with sinus of valsalva aneurysm: a case report," Journal of Cardiothoracic Surgery, vol. 3, article 46, 2008.

[3] B. A. Brown-Elliott and R. J. Wallace Jr., "Infections caused by nontuberculous mycobacteria," in Principles and Practice of Infectious Diseases, Elsevier Churchill Livingstone, Philadelphia, Pa, USA, 7th edition, 2010.

[4] S. L. Narasimhan and T. W. Austin, "Prosthetic valve endocarditis due to Mycobacterium fortuitum," Canadian Medical Association Journal, vol. 119, no. 2, pp. 154-155, 1978.

[5] R. G. Norenberg, G. K. Sethi, S. M. Scott, and T. Takaro, "Opportunistic endocarditis following open-heart surgery," Annals of Thoracic Surgery, vol. 19, no. 5, pp. 592-604, 1975.

[6] J. Olalla, M. Pombo, J. M. Aguado et al., "Mycobacterium fortuitum complex endocarditis-case report and literature review," Clinical Microbiology and Infection, vol. 8, no. 2, pp. 125129, 2002.

[7] D. Vuković, V. Parezanović, B. Savić et al., "Mycobacterium fortuitum endocarditis associated with cardiac surgery, Serbia," Emerging Infectious Diseases, vol. 19, no. 3, pp. 517-519, 2013.

[8] J. Natsag, Z. Min, Y. Hamad, B. Alkhalil, A. Rahman, and R. Williams, "A mysterious gram-positive rods," Case Reports in Infectious Diseases, vol. 2012, Article ID 841834, 4 pages, 2012.

[9] S. P. Collison and N. Trehan, "Native double-valve endocarditis by Mycobacterium fortuitum following percutaneous coronary intervention," Journal of Heart Valve Disease, vol. 15, no. 6, pp. 836-838, 2006.

[10] M. Singh, A. Bofinger, G. Cave, and P. Boyle, "Mycobacterium fortuitum endocarditis in a patient with chronic renal failure on hemodialysis," Pathology, vol. 24, no. 3, pp. 197-200, 1992.

[11] D. W. Spell, J. G. Szurgot, R. W. Greer, and J. W. Brown III, "Native valve endocarditis due to Mycobacterium fortuitum biovar fortuitum: case report and review," Clinical Infectious Diseases, vol. 30, no. 3, pp. 605-606, 2000.

[12] M. T. Kuruvila, P. Mathews, M. Jesudason, and A. Ganesh, "Mycobacterium fortuitum endocarditis and meningitis after balloon mitral valvotomy," Journal of Association of Physicians of India, vol. 47, no. 10, pp. 1022-1023, 1999.

[13] W.-J. Koh, O. J. Kwon, K. Jeon et al., "Clinical significance of nontuberculous mycobacteria isolated from respiratory specimens in Korea," Chest, vol. 129, no. 2, pp. 341-348, 2006.

[14] L. E. Bermudez, F. J. Sangari, P. Kolonoski, M. Petrofsky, and J. Goodman, "The efficiency of the translocation of Mycobacterium tuberculosis across a bilayer of epithelial and endothelial cells as a model of the alveolar wall is a consequence of transport within mononuclear phagocytes and invasion of alveolar epithelial cells," Infection and Immunity, vol. 70, no. 1, pp. 140146, 2002.

[15] L. Hall-Stoodley and H. Lappin-Scott, "Biofilm formation by the rapidly growing mycobacterial species Mycobacterium fortuitum," FEMS Microbiology Letters, vol. 168, no. 1, pp. 77-84, 1998. 


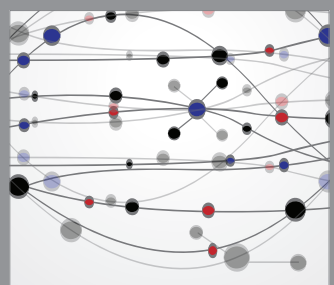

The Scientific World Journal
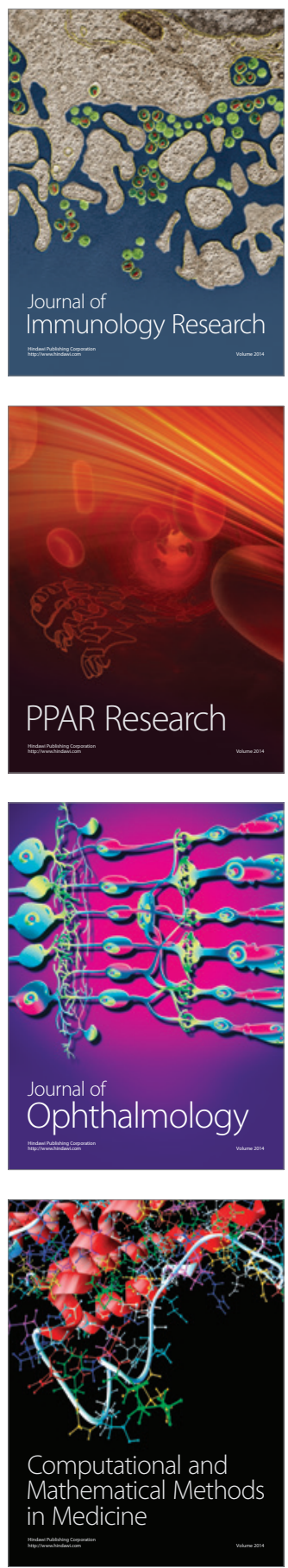

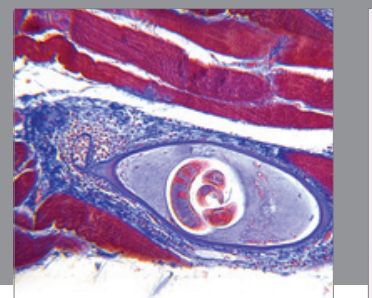

Gastroenterology

Research and Practice
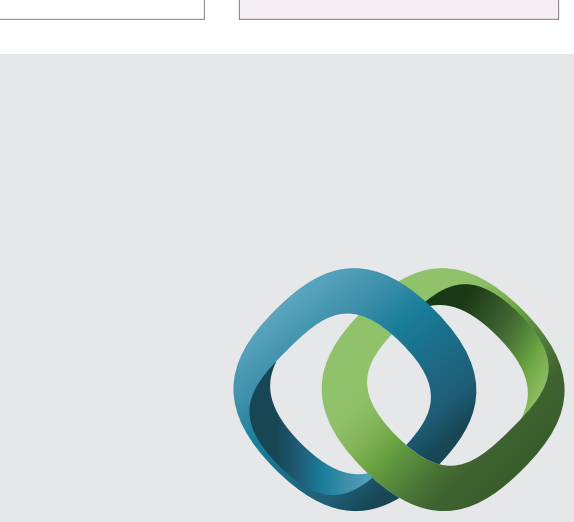

\section{Hindawi}

Submit your manuscripts at

http://www.hindawi.com
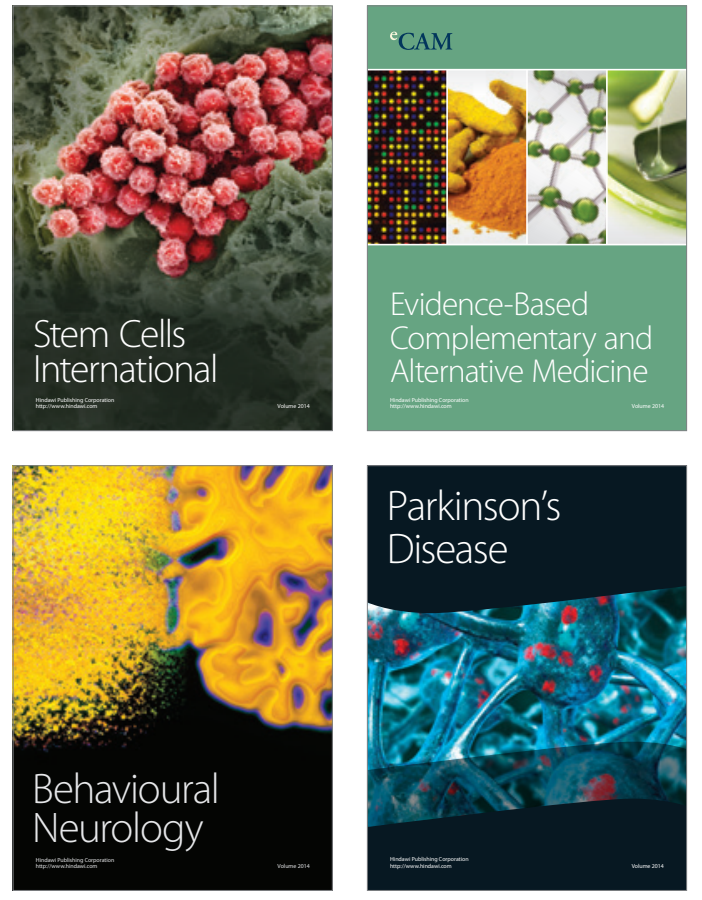
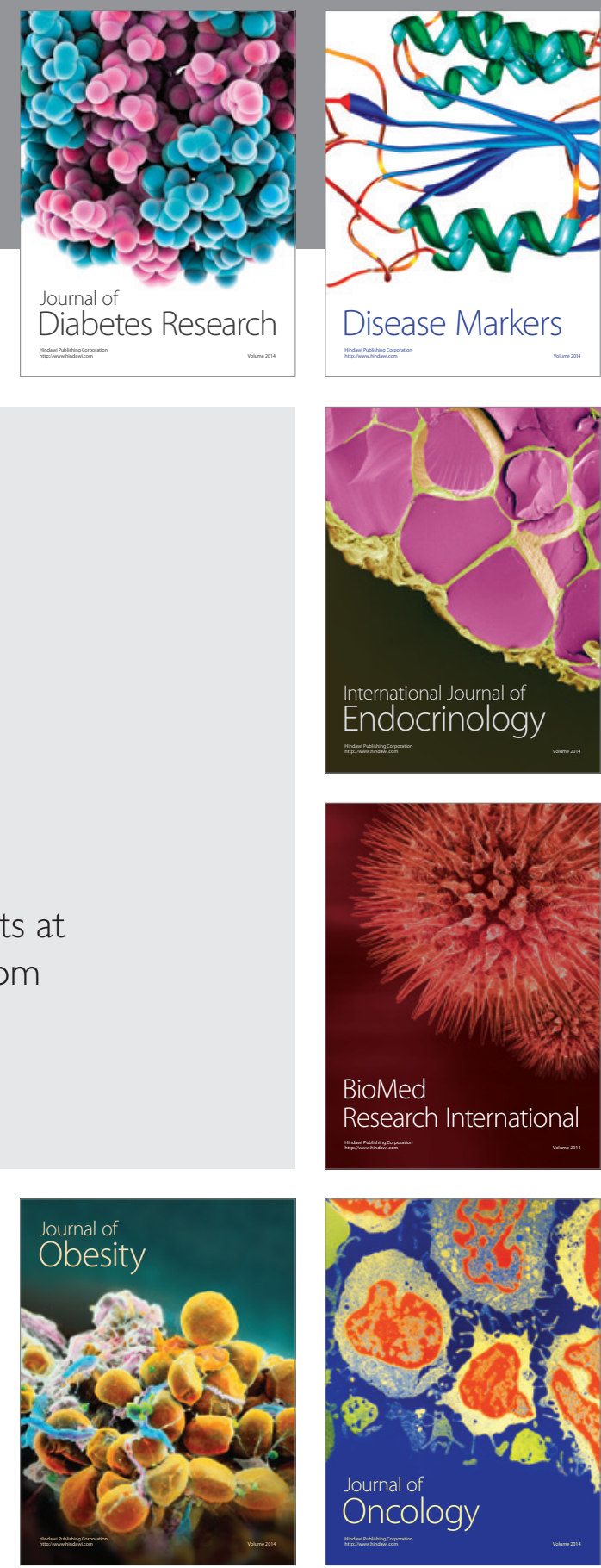

Disease Markers
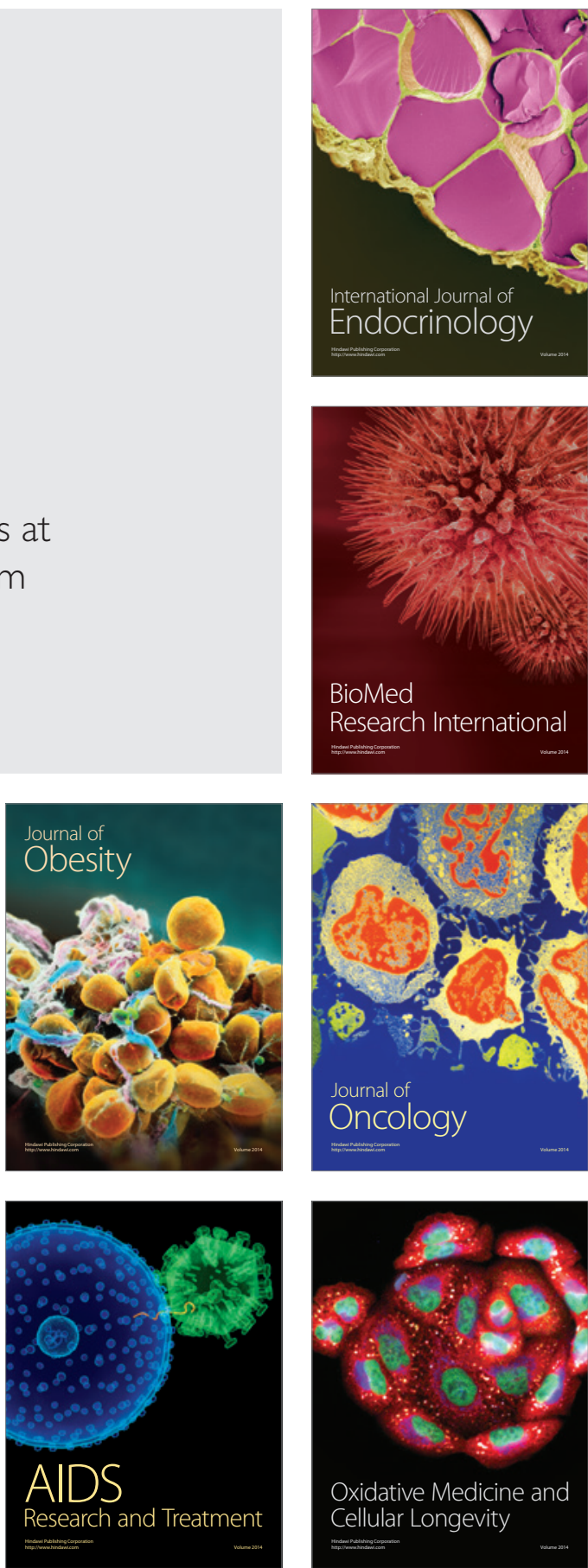